\title{
Adolescent Alcohol Exposure Reduces Behavioral Flexibility, Promotes Disinhibition, and Increases Resistance to Extinction of Ethanol Self-Administration in Adulthood
}

\author{
Justin T Gass*', William Bailey Glen Jr', Justin T McGonigal', Heather Trantham-Davidson', \\ Marcelo F Lopez ${ }^{2}$, Patrick K Randall ${ }^{2}$, Richard Yaxley ${ }^{3}$, Stan B Floresco ${ }^{4}$ and L Judson Chandler ${ }^{1,2}$ \\ 'Department of Neurosciences, Medical University of South Carolina, Charleston, SC, USA; ${ }^{2}$ Department of Psychiatry, Medical University of \\ South Carolina, Charleston, SC, USA; ${ }^{3}$ Department of Psychiatry, University of North Carolina, Chapel Hill, NC, USA; ${ }^{4}$ Department of Psychology \\ and Brain Research Centre, University of British Columbia, Vancouver, BC, Canada
}

The prefrontal cortex (PFC) is a brain region that is critically involved in cognitive function and inhibitory control of behavior, and adolescence represents an important period of continued PFC development that parallels the maturation of these functions. Evidence suggests that this period of continued development of the PFC may render it especially vulnerable to environmental insults that impact PFC function in adulthood. Experimentation with alcohol typically begins during adolescence when binge-like consumption of large quantities is common. In the present study, we investigated the effects of repeated cycles of adolescent intermittent ethanol (AlE) exposure (postnatal days 28-42) by vapor inhalation on different aspects of executive functioning in the adult rat. In an operant setshifting task, AlE-exposed rats exhibited deficits in their ability to shift their response strategy when the rules of the task changed, indicating reduced behavioral flexibility. There were no differences in progressive ratio response for the reinforcer suggesting that AlE did not alter reinforcer motivation. Examination of performance on the elevated plus maze under conditions designed to minimize stress revealed that AIE exposure enhanced the number of entries into the open arms, which may reflect either reduced anxiety and/or disinhibition of exploratory-like behavior. In rats that trained to self-administer ethanol in an operant paradigm, AlE increased resistance to extinction of ethanol-seeking behavior. This resistance to extinction was reversed by positive allosteric modulation of $\mathrm{mGluR5}$ during extinction training, an effect that is thought to reflect promotion of extinction learning mechanisms within the medial PFC. Consistent with this, CDPPB was also observed to reverse the deficits in behavioral flexibility. Finally, diffusion tensor imaging with multivariate analysis of 32 brain areas revealed that while there were no differences in the total brain volume, the volume of a subgroup of regions (hippocampus, thalamus, dorsal striatum, neocortex, and hypothalamus) were significantly different in AlE-exposed adults compared with litter-matched Control rats. Taken together, these findings demonstrate that binge-like exposure to alcohol during early to middle adolescence results in deficits in PFC-mediated behavioral control in adulthood.

Neuropsychopharmacology (20I4) 39, 2570-2583; doi:I0.I038/npp.2014.I09; published online II June 20I4

\section{INTRODUCTION}

Adolescence is a developmental period during which there is an increased drive for independence and risky decisionmaking frequently leading to experimentation with alcohol and other drugs of abuse (Casey and Jones, 2010). This heightened risk taking and impulsive decision-making are thought to be attributable, in large part, to the delayed development of the prefrontal cortex (PFC) as part of an executive control network. The PFC integrates and processes signals from cortical and subcortical structures and then uses that information to direct purposeful responses that

*Correspondence: Dr J Gass, Department of Neurosciences, Medical University of South Carolina, 67 President Street, Charleston, SC 29425, USA, Tel: +803 479 5396, Fax: 8437927353 , E-mail: gass@musc.edu

Received 4 November 2013; revised I May 2014; accepted 2 May 2014; accepted article preview online 13 May 2014 reflect both current and future circumstances (Abernathy et al, 2010). These responses include behaviors that are advantageous to the individual, but also suppression of actions that pose undue risk or harm. These processes include, among others, planning, problem solving, working memory, cognitive flexibility, and cognitive control.

Neurodevelopmental changes in the PFC during adolescence involve marked alterations in structure and connectivity (Rakic et al, 1994). Cortical gray matter volume of the PFC follows an inverted U-shaped trajectory that peaks during adolescence and declines thereafter (Blakemore and Robbins, 2012). This reduction in volume is likely due to reductions in the number of excitatory synapses via experience-dependent pruning of dendritic spines (Petanjek et al, 2011). This structural remodeling and refinement of neural circuits occurs in parallel with the maturation of the executive function of the PFC and top-down inhibitory control of behavior. 
According to the National Institute on Alcohol Abuse and Alcoholism, ethanol is the most widely consumed drug among adolescents in the United States, far outpacing the use of all other illicit drugs combined (Witt, 2010). Enhanced drive to explore, take risks, and experiment with novel activities may contribute to the increase in ethanol use during adolescence (Chambers et al, 2003). In addition to the safety concerns and adverse effects that alcohol consumption may cause during adolescence, another important but poorly understood issue is the impact of adolescent alcohol abuse on the adult brain and behavior. Alcohol consumption during adolescence frequently occurs in repeated binge-like episodes that involve large amounts of alcohol consumed over relatively short periods of time resulting in high and often dangerous levels of blood alcohol (McQueeny et al, 2009). The fact that alcohol abuse during this period is concurrent with the critical period of continued structural remodeling and refinement of PFC circuitry has led to the idea that adolescent exposure may alter PFC development and disrupt behavioral control and decision making in adulthood (Spear, 2000). While clinical studies appear to support this, such studies involving human subjects are confounded by the possibility that deficits observed in adults who abused alcohol during adolescence represent a preexisting phenotype. Therefore, well-controlled studies in animal models are needed in order to more fully understand how alcohol abuse during adolescence impacts behavioral control and decision making in adulthood. In the present study, we employed a rat model of adolescent binge-like alcohol exposure by vapor inhalation and examined alterations in PFC-dependent cognitive processes in the adult. The results of our studies are consistent with the suggestion that adolescent alcohol abuse results in changes in the cognitive function of the adult PFC. We further show that these cognitive deficits can be reversed by administration of a putative cognitiveenhancing agent when administered immediately prior to behavioral testing. This compound, 3-cyano- $N-(1,3-$ diphenyl-1H-pyrazol-5-yl)benzamide (CDPPB), is a positive allosteric modulator of the type 5 metabotropic glutamate receptor (mGluR5) and has been shown to have procognitive effects in other behavioral tasks (Ayala et al, 2009; Horio et al, 2013; Stefani and Moghaddam, 2010; Uslaner et al, 2009).

\section{MATERIALS AND METHODS}

\section{Animals}

Male (Long-Evans) rats were obtained from an in-house breeding colony with breeder stocks obtained from Charles River Laboratories. A total of 75 male Long-Evans rats were trained in the set-shifting paradigm to assess the effects of AIE on behavioral flexibility. Of those, nine rats (four AIE, five Control) were removed from the study because they failed to successfully acquire the first stage of the task that required them to learn an operant response based on the location of the light stimulus. Thus, a total of 66 rats were used for statistical analysis in the behavioral flexibility task. A subset of 22 rats were tested in the progressive ratio paradigm to assess the effects of AIE on reinforcer motivation, and a subset of 44 rats were tested for exploratory drive on the elevated plus maze (EPM). Two of these rats were removed due to erratic behavior (immediate freezing upon placement into the EPM maze), leaving a total of 42 rats for statistical analysis for the EPM. A separate group of 41 rats were used to assess the effects of AIE on ethanol self-administration and extinction learning. A total of four were removed from the study prior to the commencement of extinction training because they failed to successfully acquire operant self-administration. The criteria for successful acquisition of ethanol self-administration was $<20 \%$ variation in the number of active lever presses across three consecutive operant drinking sessions. A minimum of 30 reinforcers per session and at least 12 sessions with $10 \%$ ethanol as the reinforcer were also required. Additional information regarding the animals and drugs is provided in the Supplementary Materials and Methods.

\section{AIE Exposure}

Upon weaning at PD21, male rats were handled for $\sim 5 \mathrm{~min} /$ day to acclimate them to the experimenter handling and manipulation and thus minimize experimenter-associated stress during the subsequent AIE exposure procedure. The AIE procedure involved four repeated cycles of binge-like intermittent ethanol exposure by vapor inhalation (Figure 1a). Each cycle consisted of 2 days of ethanol exposure followed by 2 days of non-exposure. Each exposure day involved $14 \mathrm{~h}$ in the vapor chambers and

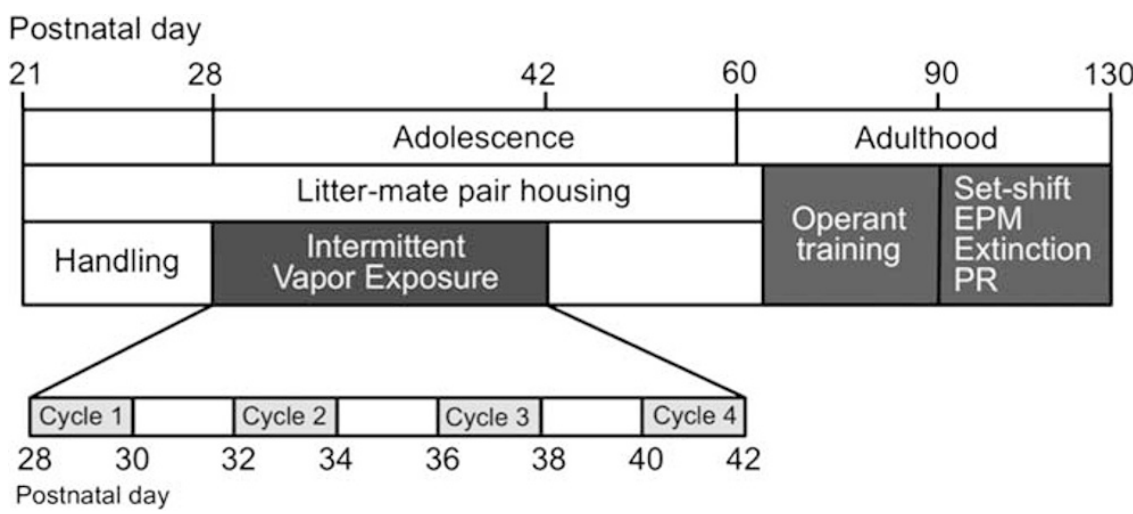

Figure I Experimental paradigm and ethanol vapor exposure model. Depicted is a schematic representation of the time line of the vapor exposure and behavioral procedures in relation to the postnatal day of the rat. 


\section{npg

$10 \mathrm{~h}$ out of the chambers. The ethanol vapor exposure chambers were constructed of clear acrylic and measured $24 \times 24 \times 14$ inches (Plas Labs; Lansing, MI). Pair-housed littermates were placed in the vapor chambers at 1800 hours and removed the following morning at 0800 hours. The light cycle in the chambers was identical to the light cycle of the rat colony room. Litter-matched Control rats were treated the same as the experimental group but were exposed to air instead of ethanol vapor. Rats remained pair-housed until their separation into single-housed rats as adults prior to the initiation of operant training procedures. Access to food and water was continuous throughout except during operant training when rats were food restricted to $\sim 90 \%$ of free-feeding weight, which escalated over time based on estimated rates of weight gain.

\section{Perfusion, Brain Preparation, and Diffusion Tensor Imaging Analyses}

Diffusion tensor imaging and image processing followed previously published procedures (Ehlers et al, 2013b). A subset of rats was killed one day after the set-shifting procedure was completed. The rats were first anesthetized with pentobarbital $(100 \mathrm{mg} / \mathrm{kg}$, intraperitoneal) and then euthanized by perfusion as described previously (Crews et al, 2004). The animals were perfused transcardially with $0.1 \mathrm{M}$ phosphate-buffered saline (PBS; $\mathrm{pH}$ 7.4), followed by 1:10 solution of Prohance (Bracco Diagnostics) in 10\% formalin. The ears and skin were then removed from the head, which was then shipped in a PBS + Prohance solution to the University of North Carolina for DTI imaging. Additional information regarding the methodology of DTI and image analysis is provided in the Supplementary Materials and Methods.

\section{Behavioral Flexibility}

Behavioral flexibility was examined using an operant setshifting procedure previously described and characterized by Floresco et al (2008). In brief, rats were first trained to respond to a light cue in order to receive a reinforcer. Once this rule was acquired, the contingencies of the task changed to a new rule that required the rat to ignore the light cue and respond only on one lever to receive a reinforcer. Behavioral flexibility was then assessed by measuring the number of trials required to learn the new rule. Previous studies have shown that shifting strategies using this operant-based assay is sensitive to inactivation or other manipulations of the medial PFC (Floresco et al, 2008; Enomoto et al, 2011), which can increase perseverative response during the shift. The primary dependent variables in these experiments were the number of completed trials required and errors made to achieve criterion performance of 10 consecutive correct choices. The trials to measure criterion did not include trials where rats did not make a response. Thus, differences in performance on these measures would be independent of any differences in the omissions rates between groups. A detailed description of this procedure is provided in the Supplementary Materials and Methods.

\section{Progressive Ratio}

The progressive ratio $(\mathrm{PR})$ paradigm was carried out in the operant boxes above to examine potential group differences in the reinforcing property of $20 \%$ sweetened condensed milk (the same reinforcer used in the behavioral flexibility experiment). In this paradigm, the number of lever presses required to obtain a single delivery of $20 \%$ SCM was determined by the following equation: responses per reinforcer delivery (rounded to the nearest integer) $=$ $\left(5 e^{(\text {reinforcer number } \times 0.2)}\right)-5(\mathrm{eg}, 1,2,4,6,9,12,15,20,25$, 32,40 , etc) where $e$ is the base of the natural log (Richardson and Roberts, 1996). All PR testing was conducted in $16 \mathrm{~h}$ overnight sessions, and each reinforcer delivery was followed by a $4 \mathrm{~s}$ timeout period during which time, additional active lever presses were recorded but produced no programmed consequences. The primary dependent variables measured during each $16 \mathrm{hr}$ session were the number of active and inactive lever presses, the number of reinforcers earned, and the break-point (defined as the max number of lever responses preceding a time when the rat failed to press the lever for $1 \mathrm{~h}$ ).

\section{Elevated Plus Maze}

Exploratory drive was examined using an EPM (http:// www.anymaze.com/index.htm) with the following dimensions: arm width, $10 \mathrm{~cm}$; arm length, $50 \mathrm{~cm}$; closed arm wall height, $40 \mathrm{~cm}$; open arm wall height, $1 \mathrm{~cm}$; maze leg height, $40 \mathrm{~cm}$. In the behavioral testing room, light was provided by two red lights located above each of the closed arms of the maze. Rats were habituated to the room in their home cage with no room lights for $2 \mathrm{~h}$ the day before testing on the maze. On the day of the test, each rat was individually transported to the testing room and left in their home cage with the test lights on for $5 \mathrm{~min}$. The rat was then placed on the maze in the central area facing an open arm and allowed to roam the maze for $5 \mathrm{~min}$ with the experimenter exiting the room during the test session. A digital video of each rat's activity was acquired for offline analysis of the following variables: time in the open $v s$ closed arms, percent entries into the open $v s$ closed arms, and total number of entries into either of the arms. Overall activity levels were tracked automatically (eg, total distance traveled) while the rats' location on the maze was scored manually for increased accuracy in one of the three mutually exclusive locations: the central area, closed arms, or open arms. Rats were considered in an open or closed arm when all four paws were within an arm. The percent entries and time spent in the open arms were calculated by dividing the amount of time spent in the open arms by the time spent in both the open and closed arms.

\section{Ethanol Self-Administration}

Beginning on PD65, rats were trained to orally selfadminister ethanol by first exposing them to an intermittent two-bottle choice drinking initiation paradigm for a period of 2 weeks (Simms et al, 2010; 2011). Three days per week, two bottles-one containing water and the other containing $20 \%$ ethanol-were placed on the cage. Twenty-four hours later, the bottles and the rats were weighed and $\mathrm{g} / \mathrm{kg}$ of 
ethanol consumed was calculated. The day following the last two-bottle choice session, the rats were placed in operant chambers (as described above) and trained to self-administer a $20 \%$ ethanol solution in $1 \mathrm{~h}$ sessions 3 days per week. Rats were trained to lever press on the active lever to receive ethanol on an FR1 schedule of reinforcement. Each active lever press activated the syringe pump to deliver $\sim 45 \mu \mathrm{l}$ of a $20 \%$ ethanol-water solution over a $1.5 \mathrm{~s}$ period. During ethanol delivery, the stimulus light above the active lever was illuminated and the tone was presented. Following each reinforcer delivery, a 4 sec timeout period was initiated during which additional active lever presses were recorded but had no programmed consequences. After stable response for $20 \%$ ethanol was reached ( $\sim 10-12$ sessions), the concentration of ethanol was reduced to $10 \%$ and the session length was shortened to $30 \mathrm{~min}$ for the remaining sessions (12-16 sessions). Following stabilization of response for $10 \%$ ethanol, blood samples $(20 \mu \mathrm{l})$ were taken from the tail vein using heparin-coated borosilicate capillary tubes immediately following a 30 min self-administration session for subsequent analysis of blood ethanol levels (see below). After an additional week of daily self-administration sessions, extinction training procedures commenced. Additional information on training for ethanol self-administration is provided in the Supplementary Materials and Methods.

\section{Extinction of Ethanol-Seeking Behavior}

Extinction of self-administration and treatment with the mGluR5-positive allosteric modulator CDPPB commenced after criteria for maintenance of ethanol self-administration were achieved. Rats from Control and AIE groups were then assigned to either CDPPB- or vehicle-treatment groups for extinction training. Thus, there were a total of four groups: AIE with vehicle treatment (AIE/Vehicle), Control with vehicle treatment (Control/Vehicle), AIE with CDPPB treatment (AIE/CDPPB), and Control with CDPPB treatment (Control/CDPPB). Extinction training was conducted in $30 \mathrm{~min}$ daily sessions in the presence of ethanol-associated cues (eg, presentation of the light/tone stimulus complex for $1.5 \mathrm{~s}$ following each active lever press, followed by a $4 \mathrm{~s}$ timeout), as it has been observed that such procedures produce drug-seeking behavior that is more resistant to extinction than that observed during extinction in the absence of drug-associated cues (Ranaldi and Roberts, 1996). No ethanol solution was delivered during extinction sessions, and presses on the inactive lever during extinction were recorded but produced no programmed consequences.

Twenty minutes prior to each extinction session, rats were administered vehicle (10\% Tween 80$)$ or CDPPB (30 $\mathrm{mg} / \mathrm{kg}$ s.c.) according to their group assignment and returned to their home cages. The dose of CDPPB was based on our previous studies (Cleva et al, 2011; Gass and Olive, 2009) as well as other studies (Kufahl et al, 2012; Reichel et al, 2011) that showed facilitation of extinction learning, procognitive effects, and no effects on motor behavior. Rats were placed in the self-administration apparatus and extinction criteria was considered to have been met when the number of active lever presses exhibited by an individual rat was $<20 \%$ (for 2 consecutive days) of those observed on the average of the last 2 days of active drug selfadministration for that particular rat.

\section{Statistical Analyses}

Behavioral data was analyzed using SPSS version 21.0 software (SPSS, Chicago, IL) and Prism version 6 (GraphPad Software, La Jolla, CA). In experiments involving analysis by ANOVAs, pairwise post hoc tests with adjustment for multiple comparisons were performed. For analysis of behavioral flexibility, a one-way ANOVA was used to compare groups on the number of trials required to reach the criterion in both visual cue and response discrimination phases. A one-way ANOVA was also used to compare the groups on the number of perseverative and regressive errors during the response discrimination phase. For the PR experiment, a two-way ANOVA was used to compare the groups on total active lever presses, total reinforcements, and average break-point during the session. For the EPM task, activity was scored in Ethovision XT 8.5 (http://www.noldus.com) and overall activity levels were tracked automatically (eg, total distance traveled). For increased accuracy, the location of the rat on the maze was scored manually in one of the three mutually exclusive locations, the central area, closed arms, or open arms. The percent entries and time spent in the open arms were calculated by dividing the amount of time spent in the open arms by the time spent in both the open and closed arms. All statistical comparisons in this task were made with Independent Samples $t$-tests.

For analysis of ethanol self-administration and extinction, active lever responses and number of reinforcements during the maintenance phase of self-administration were examined using Independent Samples $t$-tests. During the extinction phase, active lever presses on the last 2 days of active self-administration of $10 \%$ ethanol (ie, maintenance) were averaged and compared with individual days of extinction training using repeated-measures ANOVA. Results from this analysis provided a statistical basis for determination of extinguished response. An Independent Samples $t$-test was used to analyze the number of sessions required to reach extinction criteria. The number of responses on the inactive lever was minimal ( $<5$ per session) and these data were not analyzed.

For the behavioral flexibility and extinction experiments involving $\mathrm{CDPPB}$, one-way ANOVAs with pairwise post hoc tests with adjustments for multiple comparisons were performed. For instance, in the behavioral flexibility experiments, this type of analysis allowed for the comparison among the four groups during the initial learning of the rule and the set shift. In addition, this analysis allowed for comparison among the four groups on the types of errors occurring during the set-shift. The same type of statistical analysis also allowed for comparison among the four groups during the multiple days of extinction as well as the number of sessions required to reach extinction criteria.

\section{RESULTS}

\section{AIE Vapor Exposure}

The present studies utilized an intermittent model of alcohol exposure by vapor inhalation during early to middle adolescence (PD28-42) that is designed to simulate repeat episodes of binge-like excessive alcohol exposure. Rats were subjected to four cycles of two consecutive episodes of 
alcohol vapor inhalation with each exposure consisting of $14 \mathrm{~h}$ in the vapor chambers followed by $10 \mathrm{~h}$ out of the chambers. Therefore, as depicted in Figure 1, rats were exposed to alcohol on PD28 and 29 (cycle 1), PD32 and 33 (cycle 2), PD36 and 37 (cycle 3), and PD40 and 41 (cycle 4). We used a 5-point behavioral intoxication rating scale (Nixon and Crews, 2002) to provide an index of the level of intoxication that was assessed at the end of each of the exposure cycles. In brief, the rats were scored according to the following behavioral scale: $1=$ no signs of intoxication; $2=$ slightly intoxicated (slight motor impairment); $3=$ moderately intoxicated (obvious motor impairment but able to walk); $4=$ highly intoxicated (dragging abdomen, loss of righting reflex); $5=$ extremely intoxicated (loss of righting reflex and loss of eye blink reflex). We chose a target level of slight-to -moderate intoxication, which corresponds to an intoxication rating of 2-3, respectively. In addition to providing a measure of the level of intoxication, the rating also provided immediate information that could be used to make adjustments to the level of ethanol vapor in the chambers. The intoxication score averaged across all four cycles for all rats was $2.71 \pm 0.05$, with an average daily median of $2.5 \pm 0.05$ (quartiles: $Q 1=2.5$; $\mathrm{Q} 2=3.0 ; \mathrm{Q} 3=2.5 ; \mathrm{Q} 4=2.0$ ). As shown in Figure $2 \mathrm{a}$, rats showed significant tolerance between the first and second day of exposure as indicated by a significant cycle $\times$ day interaction $[\mathrm{F}(3,304)=7.08, p=0.0001]$. Post hoc analyses revealed that there was a significant difference between day 1 and day 2 for the first three cycles ( $p$ values $<0.05$, indicated by $\left.{ }^{*}\right)$. However, no difference in the level of intoxication was observed in the fourth cycle $(p=0.99)$. Furthermore, analysis of the intoxication rating for day 1 of each of the four cycles also revealed significant differences among the days $[\mathrm{F}(3,203)=17.94, p<0.0001]$. Post hoc analyses showed that the intoxication rating for day 1 of cycle 2 was significantly increased compared with cycle 1 $(p=0.03$, indicated by $\#)$. In addition, the intoxication rating for day 1 of cycle 4 was significantly decreased compared with cycle $1 \quad(p=<0.0001$, indicated by \#\#). Intoxication ratings across exposures were also analyzed as a within-subject (exposure by day) design using a generalized estimating equation (GEE) analysis for a logistic ordinal response to accommodate the repeated, ordinal category values. A highly significant interaction $\left(\chi^{2}(3)=77.4\right.$, $p<0.0001$ ) of day by exposure period was detected by GEE analysis. Overall intoxication was rated more highly on the first, as opposed to second day $\left(\chi^{2}(1)=21.54, p<0.0001\right)$. Simple main effects of day within exposure period revealed significant day $1 v s$ day 2 effects on the first three exposures (all $p$ values $<0.0001$ ) but not on the fourth exposure period $\left(\chi^{2}(1)=0.325, p=0.569\right)$. Together, this analysis indicates tolerance development not only within the cycles but also across the cycles of exposure.

As a complementary measure to the behavioral rating of intoxication, at the end of each four exposure cycles, we also collected tail vein blood and determined the BEC. This revealed average BEC values (in $\mathrm{mg} \%$ ) of $310.22 \pm 15.02$, $357.39 \pm 24.3,358.49 \pm 14.88$, and $288.24 \pm 17.5$, respectively (Figure 2b), and an overall grand average across all four cycles of $328.58 \pm 9.4 \mathrm{mg} \%$. As expected, a comparison of BECs using both tail and trunk blood (obtained from the same rat; PD39) showed nearly identical values indicating that tail vein blood accurately reflects BEC in the adolescent rat (Figure 2c). As shown in Figure 2d, analysis using GEE revealed that the intoxication rating scale behaved well with increasing BEC associated with increasing ratings for day 2 (BEC were not measured after day 1 ). There was no difference in the response function for exposure 1 and 4 $\left(\chi^{2}(1)=0.996, p=0.326\right)$ and was highly significant across exposures $\left(\chi^{2}(1)=23.2, p<0.001\right)$. Finally, analysis of body weights at the end of experimental testing showed no significant difference between Control and AIE-exposed rats $[\mathrm{t}(95)=0.16, p=0.87]$.

\section{Diffusion Tensor Imaging Assessment of Brain Regional Volume}

To investigate the effect of AIE exposure on gross brain morphology in the adult, we acquired postmortem DTI of brains from a group of Control $(n=6)$ and AIE-exposed $(n=7)$ rats. Using a recently published $3 \mathrm{D}$ imaging atlas (Rumple et al, 2013), we obtained fractional anisotropy, diffusion, and volume measurements for 32 brain regions (Supplementary Table S1) As the original 3D atlas of 29 brain regions did not breakout the medial PFC and orbitofrontal cortex from the neocortex or the striatum from the midbrain, we modified the atlas based upon the boundaries in the 2D atlas of Paxinos and Watson (2005) to include these areas as separate distinct regions. As there is the potential for false positives (type 1 error) when performing a large number of tests across data sets (eg, 32 different brain regions), we analyzed the data using a partial least squares (PLS) approach. In this multivariate technique, the set of predictors (in this case, regional volumes corrected for total brain volume) is factored into a set of latent variables in as much the same way as principle components or factor analysis is performed. However, in contrast to those techniques, PLS finds the latent variables that optimally predict one or a set of dependent variables rather than the set that predicts the maximal variance among the predictors. Thus variables may be detected that, while not significantly predicting the outcome alone, are closely associated with latent variables that do predict the outcome. In addition, latent variables that explain significant variance in the predictors are ignored if they are unrelated to the outcome. PLS discriminative analysis (PLS-DA) is a variant that is optimized for predicting dichotomous outcomes, like group membership (SOLO, Eigenvector Research).

Preliminary examination of the variance suggested that a two-factor model (two latent variables among the regional measurements) was optimal in separating the groups with the first being of substantially greater importance. Two indices of the overall importance of each region in predicting group (Control vs AIE) membership were examined. In both cases, higher values of the variable importance (VIP) and the selection ratio (SR) indicate greater importance in the prediction. Figure 3 shows the plot of the VIP values $v s$ the SR for the 32 different regions in the overall model. Five regions significantly contributed to the model prediction as shown in the cluster of regions in the upper right of the figure (also see Supplementary Figure S1). These five regions also had significant loadings on the first latent variable. The two-factor model successfully assigned 


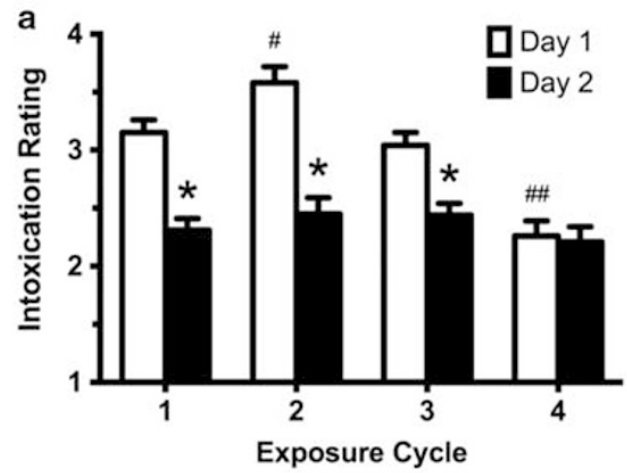

b
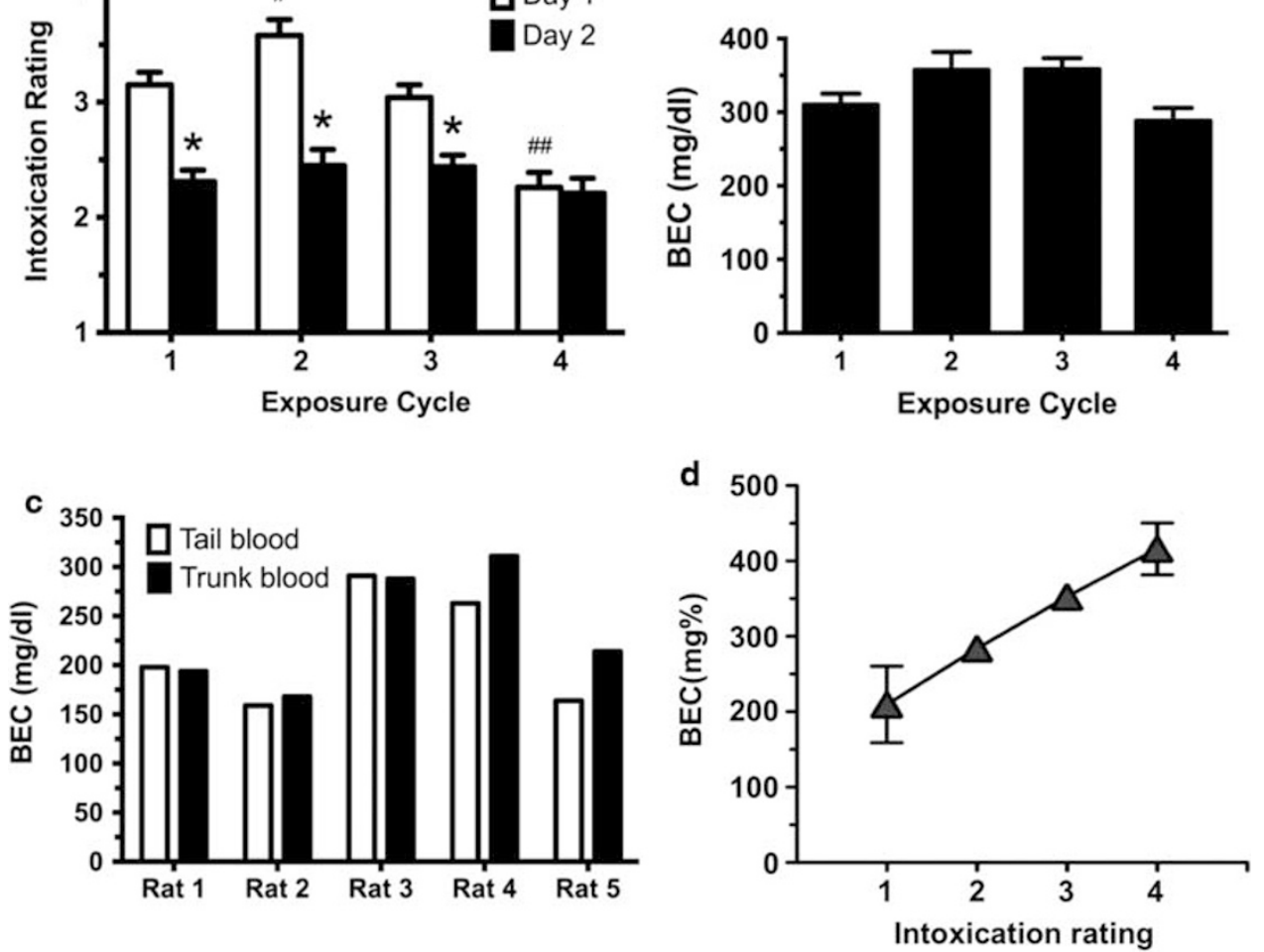

Figure 2 Characterization of the level of intoxication and BEC of the adolescent intermittent ethanol (AIE) vapor exposure procedure. (a) Comparison of the level of intoxication in rats at the end of each cycle of ethanol exposure by vapor inhalation. Immediately after removal from the vapor chambers, rats were assessed for their level of behavioral intoxication using an intoxication rating of $1-5$. The target level of intoxication was a rating of 2 (slight motor impairment) to 3 (moderate motor impairment). Shown are the average \pm SEM of intoxication rating at each cycle of exposure. *Indicates significant decrease $(p<0.05)$ from day I of cycles I-3; \# indicates significant increase $(p<0.05)$, and \#\# indicates significant decrease $(p<0.00$ I $)$ from day I of cycle I-3. (b) BEC values were obtained from tail vein blood drawn immediately following chamber exposure on days 3, 7, II, and I5. Shown on the graph are the mean \pm BEC for each of the four cycles. (c) Comparison of BEC levels from tail and trunk blood obtained from the same rat revealed they are highly consistent between the two compartments. (d) The level of intoxication based upon the intoxication rating scale strongly correlated with the BEC. Shown is the BEC (mean \pm SEM) combined from AIE cycles one and four taken at the end of day 2 of each cycle. Statistical analysis revealed a highly significant correlation of the $\mathrm{BEC}$ reading with the level of intoxication $(p=0.00 \mathrm{I})$.

the correct case to all except one rat. Repeated estimations of the model dropping one rat each time performed equally as well, especially when the outlying animal was dropped. As can be seen in Figure 3, a group of brain areas (hippocampus, thalamus, dorsal striatum, neocortex, and hypothalamus) clearly separate as significant contributors to the model. Nominal tests of the VIP over its bootstrap error resulted in $p<0.01$ in all five regions. A reduced model using only those regions predicted nearly as well as the full model (misassigning two rats, rather than one). Of the brain regions that were identified as significantly contributing to the model, four of the five were reduced in volume $\left(\mathrm{mm}^{3}\right)$ as follows (Control vs AIE, \% reduction): hippocampus, $89.25 \pm 1.42$ vs $82.82 \pm 2.34$ ( $-7.2 \%$ ); thalamus, $49.39 \pm 1.12$ vs $43.25 \pm 1.98$ ( -12.4$)$; striatum, $75.69 \pm$ 1.23 vs $69.19 \pm 2.54$ (-8.6); neocortex, $536.15 \pm 5.21$ vs $510.23 \pm 10.54(-4.8)$. In contrast, the mean volume of the hypothalamus was increased in the AIE-exposed adult rats $(53.59 \pm 0.93$ vs $55.63 \pm 1.55(+3.8 \%)$. Wet weight of the whole brain obtained after removal of the brains from the skull following imaging, revealed there was no significant difference between Control $(1.62 \mathrm{~g} \pm 0.03)$ and AIE $(1.55 \mathrm{~g} \pm$ $0.04)[\mathrm{t}(11)=1.45, p=0.1751]$. This is consistent with the
DTI measurement of total brain volume that also revealed no significant difference between Control (2075.8 \pm $\left.18.0 \mathrm{~mm}^{3}\right)$ and AIE $\left(2025.2 \pm 32.5 \mathrm{~mm}^{3}\right),(-2.4 \%)$. These exploratory findings using DTI imaging of the intact rat whole brain suggest that AIE exposure may result in pathological changes to a subset of brain regions in the adult that have been implicated in cognition and addiction.

\section{Behavioral Flexibility}

To assess whether AIE exposure altered behavioral flexibility in adulthood, we utilized an operant set-shifting task that requires the rat to alter its behavior in response to a rule change. Rats were initially trained on a rule that required them to choose the lever indicated by a stimulus light to receive a reinforcer. Rats from both groups required 3-5 daily sessions to acquire the first rule, and there was no difference between Control and AIE rats in the number of sessions required to learn this rule (Supplementary Figure $\mathrm{S} 2 \mathrm{~A}$ ) or in the total number of omissions during the learning of the rule (Table 1). As shown in Figure 4a, on the final day of training for the initial rule, the number of trials required to reach criterion on the this rule were equal 


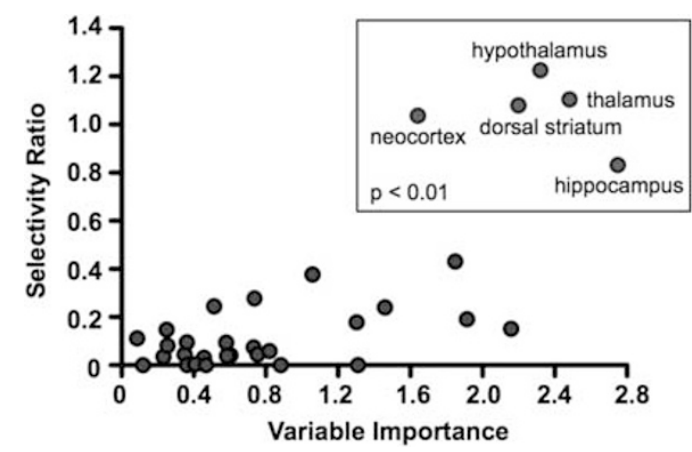

Figure 3 Adolescent alcohol exposure results in alterations in volume of a subset of brain regions in the adult rat assessed by diffusion tensor imaging (DTI). Volumetric measurements of 32 different regions were subjected to partial least squares discriminative analysis (PLS-DA) from which the selectivity ratio (SR) and variable importance (VIP) values were obtained. As shown in the figure, plotting the SR versus VIP resulted in a group of five brain regions that are clearly separate (boxed area). Nominal tests of VIP over its bootstrap error revealed significance $(p<0.01)$ for all five of these brain regions. The brain areas that were included in the analysis were as follows: amygdala, anterior commissure, anterior piriform, aqueduct, brainstem, central gray, cerebellum, corpus callosum, entorhinal cortex, external capsule, fimbria, fornix, fourth ventricle, genu, hippocampus, hypothalamus, inferior colliculus, internal capsule, lateral ventral, medial prefrontal cortex, neocortex, olfactory bulb, orbital cortex, rest of forebrain, rest of midbrain, splenium, striatum, substantia nigra, superior colliculus, thalamus, third ventricle, ventral tegmental area.

Table I Mean $( \pm$ SEM) Number of Trial Omissions Made During the Visual Cue Discrimination and Strategy Shift to a Response Rule Phases of the Set-Shifting Tasks

\begin{tabular}{lcc}
\hline Treatment & Visual cue & Shift to response \\
\hline Controls & $31.86( \pm 18)$ & $1.52( \pm 1)$ \\
AlE & $30.8( \pm I 2)$ & $4.96( \pm I)$ \\
Control + saline & $2.4( \pm 1)$ & $2.1( \pm 2)$ \\
AlE + saline & $2.0( \pm 1)$ & $6.4( \pm 1)$ \\
Control + CDPBB & $2.56( \pm 1)$ & $4.1( \pm 2)$ \\
AlE + CDPBB & $2.0( \pm I)$ & $1.6( \pm 1)$ \\
\hline
\end{tabular}

${ }^{a} p<0.05$ vs Controls.

between the Control and AIE groups indicating that there was no effect of AIE on the visual cue discrimination phase of the task (Control, $n=23$; AIE, $n=24 ; \mathrm{t}(89)=0.038$, $p=0.991)$. However, when tested for their ability to shift their response strategy, AIE-exposed rats required significantly more trials to shift from the visual cue rule to the location rule $[\mathrm{t}(45)=2.850, p=0.006]$ (Figure 4a). In addition, AIE rats showed a significant increase in the total number of errors $[\mathrm{t}(45)=2.02, p=0.04]$ (Figure $4 \mathrm{~b}$ ). Finally, AIE-exposed rats showed a slight, but statistically significant increase in the number of omissions during the set-shift $\left[\mathrm{t}(45)=3.330,{ }^{\star} p=0.0009\right]$ (Table 1$)$. These data indicate that AIE rats had more difficulty relative to Control rats with updating their behavioral strategy in response to the change in reinforcer contingency.
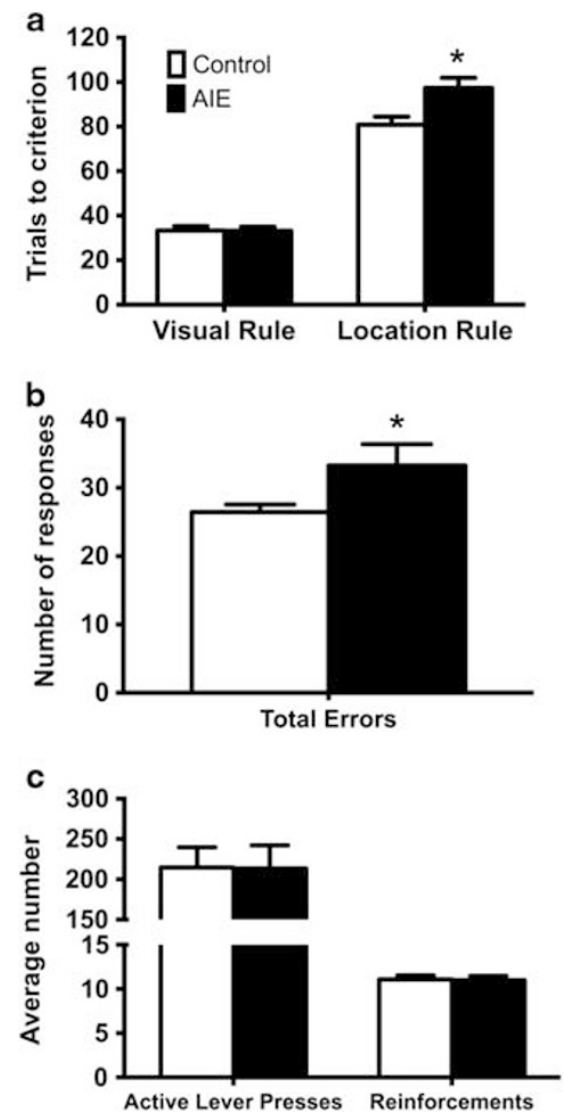

Figure 4 AlE exposure results in deficits in performance on an operant set-shifting task in adulthood. (a) There were no differences between the groups in the number of trials required to reach criteria on the first rule. However, when tested for their ability to switch to the new rule (location rule), AlE-exposed rats required more trials to reach criterion. (b) The increase in the number of trials required to shift to the new rule was associated with a significant increase in the number of total errors. (c) Control and AlE-exposed rats showed equal reinforcer motivation when tested on a progressive ratio responding procedure. ( ${ }^{*} p<0.05$ vs Control; Control, $n=13 ;$ AlE, $n=14$ ).

To determine whether AIE altered the rats' motivation to obtain the reinforcer, we next examined PR response in a subset of rats following testing of behavioral flexibility. As shown in Figure 4c, there were no differences in active lever presses between Control- and AIE-exposed rats $[\mathrm{F}(1,42)=0.998, p=0.98]$, indicating that AIE did not alter motivation to obtain the reinforcer during performance of the set-shifting task. Analysis of the time course data using cumulative records also shows that both Control and AIE rats exhibit similar response patterns for the reinforcer (Supplementary Figure S3). Collectively, the results of these two experiments demonstrate that AIE exposure does not impair discrimination learning nor does it induce nonspecific motivational deficits. However, this treatment leads to pronounced deficits in behavioral flexibility in adulthood.

In light of the above observation, we next examined the ability of CDPPB - an mGluR5-positive allosteric modulator (PAM) with cognitive-enhancing properties-to reverse the behavioral inflexibility associated with AIE exposure. Prior to initiation of training on this task, rats were assigned to 
one of the four groups: AIE with vehicle treatment (AIE/ Vehicle; $n=10$ ), Control with vehicle treatment (Control/ Vehicle; $n=8$ ), AIE with CDPPB treatment (AIE/CDPPB; $n=10$ ), or Control with CDPPB treatment (Control/CDPPB, $n=11)$. First, there were no differences among the groups in the number of daily sessions required to learn the visual cue rule (Supplementary Figure $2 \mathrm{~b}$ ) or in the total number of omissions during the learning of the rule (Table 1). As shown in Figure 5, there were no differences among these four groups in the number of trials required to reach criterion on this rule indicating that there were no effects of either AIE on the visual cue discrimination phase of the task as all rats learned to perform this task to criterion within 30 trials (Figure 5a). However, when tested for their ability to shift response strategy to the location rule, there were significant differences among the groups $[\mathrm{F}(3,35)=6.894$, $p=0.0009]$ (Figure 5a). AIE rats required significantly more trials to learn the response discrimination rule compared with the Control group, replicating the effect observed in our previous experiment $(p=0.035)$. Interestingly, treatment with CDPPB prior to the set-shift completely reversed the deficits in behavioral flexibility observed in AIE rats $(p=0.001)$ without altering response in the Control group of animals. For this set of experiments, we modified the output of the program such that we were now able to determine the type of error being made (which we were not
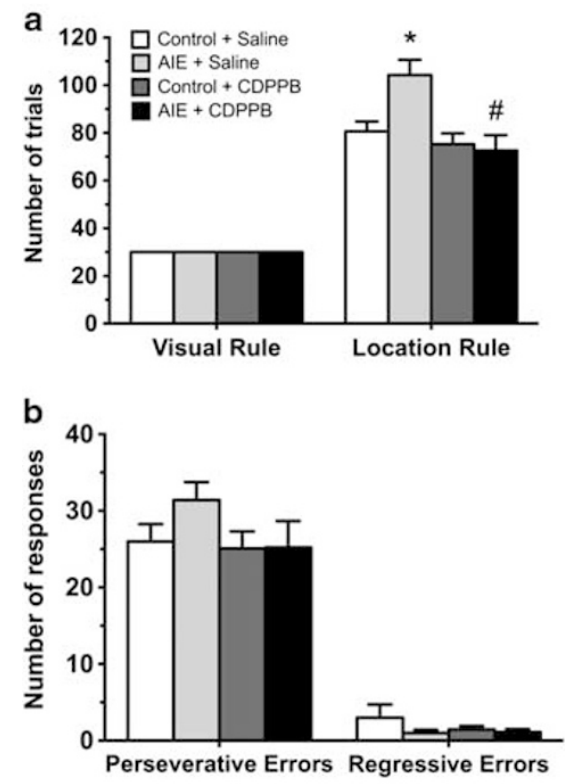

Figure 5 Administration of CDPPB immediately prior to testing in the operant set-shifting task attenuates AIE exposure-induced deficits in behavioral flexibility in adulthood. (a) There were no differences between the groups in the number of trials required to reach criteria on the first (visual) rule (all rats learned to perform this task within 30 trials). When tested for their ability to switch to the new rule (location), AIE + vehicletreated rats required more trials to reach criterion ( $p<0.05$ compared with all other groups). Treatment with CDPPB prior to the set-shift significantly reduced the number of trials required for criterion in AIEexposed rats to a level similar to Control rats $\left.{ }^{\#} p<0.05\right)$. (b) Although $\mathrm{AlE}+$ saline rats displayed more perseverative errors compared with all other groups, these increases were not significantly different and there were no differences among the groups in total regressive errors during the set-shift procedure (AIE and Controls, $n=10$ ). able to do in the initial set of studies shown in Figure 4). A perseverative error was defined as an incorrect response that occurred prior to a rat making five correct responses in a row for the first time during the session. All subsequent incorrect responses were labeled as regressive errors. Although AIE rats had more perseverative errors compared with the other groups during the shift to response discrimination, this increase was not statistically significant $[\mathrm{F}(3$, $37)=1.304, p=0.288$ ] (Figure 5b). In addition, there were no significant differences among the groups in the number of regressive errors $[\mathrm{F}(3,35)=1.35, p=0.274]$ (Figure $5 \mathrm{~b}$ ) or the number of omissions $[\mathrm{F}(3,37)=2.381, p=0.085]$ (Table 1). Unlike the statistical analyses for a two-group comparison shown in Figure 4, a different statistical approach was required to compare these four groups (ANOVA) and thus, this reduction in power likely contributed to the nonsignificant findings in error types. Regardless, these results indicate that while AIE exposure resulted in deficits in the ability of adult rats to shift their strategy in response to changes in reinforcer contingency, modulation of mGluR5 activity immediately prior to behavioral testing can reverse this deficit.

\section{Performance on the EPM}

After completion of the behavioral flexibility and PR tasks, a subset of rats was tested on the EPM (Control: $n=20$; AIE: $n=22$ ). As shown in Figure 6 a, AIE rats spent significantly more time in open arms compared with the closed arms $[\mathrm{t}(39)=2.524, p=0.0158)$. There was also a significant increase in percent entries into the open $v s$ closed arms in AIE-exposed rats compared with Control rats $[\mathrm{t}(39)=3.278$, $p=0.002$ ] (Figure 6b). However, there was no significant difference between the groups in total number of entries into either of the arms (Figure 6c). These results suggest

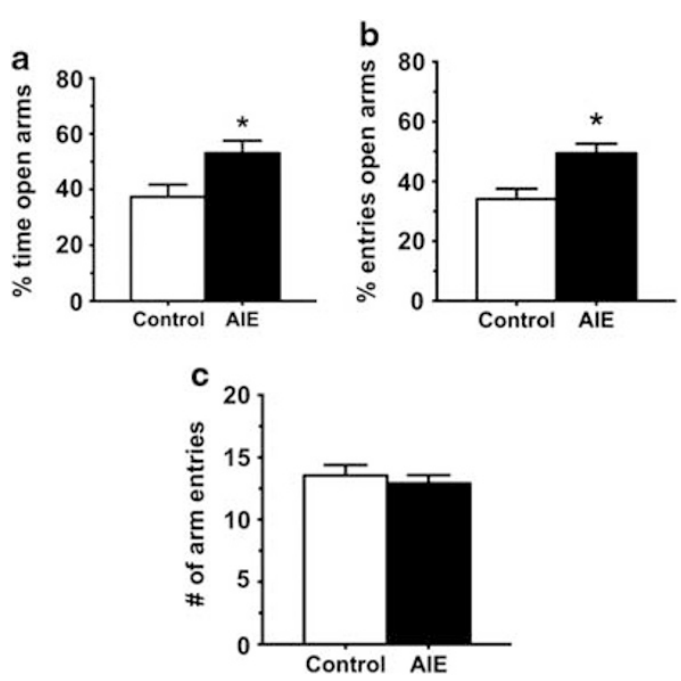

Figure 6 Adult AlE-exposed rats exhibit reduced anxiety and/or disinhibitory-like behavior on the elevated plus maze. (a) AlE exposure resulted in a significant increase in percent time in the open arms compared with Controls ( $p<<0.05$ vs Control). (b) There was also a significant increase in the percent entries into the open arms in AlE-exposed rats $\left({ }^{*} p<0.05\right.$ vs Control). (c) There were no differences in the total number of arm entries between AIE and Control rats ( $p>0.05$; Control, $n=20$; AIE, $n=22$ ). 
that AIE exposure reduced anxiety and/or reduced inhibition of exploratory behavior in adulthood.

\section{Ethanol Self-Administration and Extinction}

In this set of studies, a separate group of rats that did not receive cognitive testing were first trained using operant procedures to self-administer alcohol and then underwent extinction training, a process that is thought to be mediated by the medial PFC (Gass and Chandler, 2013; Peters et al, 2009). After the establishment of stable baseline operant responding for a $20 \%$ solution of ethanol, adult rats that had undergone AIE exposure exhibited enhanced levels of ethanol self-administration in comparison with Control rats (Figure 7a). This was observed as an increase in the mean number of lever presses $[\mathrm{t}(36)=3.110, p=0.003]$ and in the number of alcohol reinforcements [ $\mathrm{t}(36)=2.952, p=0.005]$ that were delivered during the 30 -min operant session.
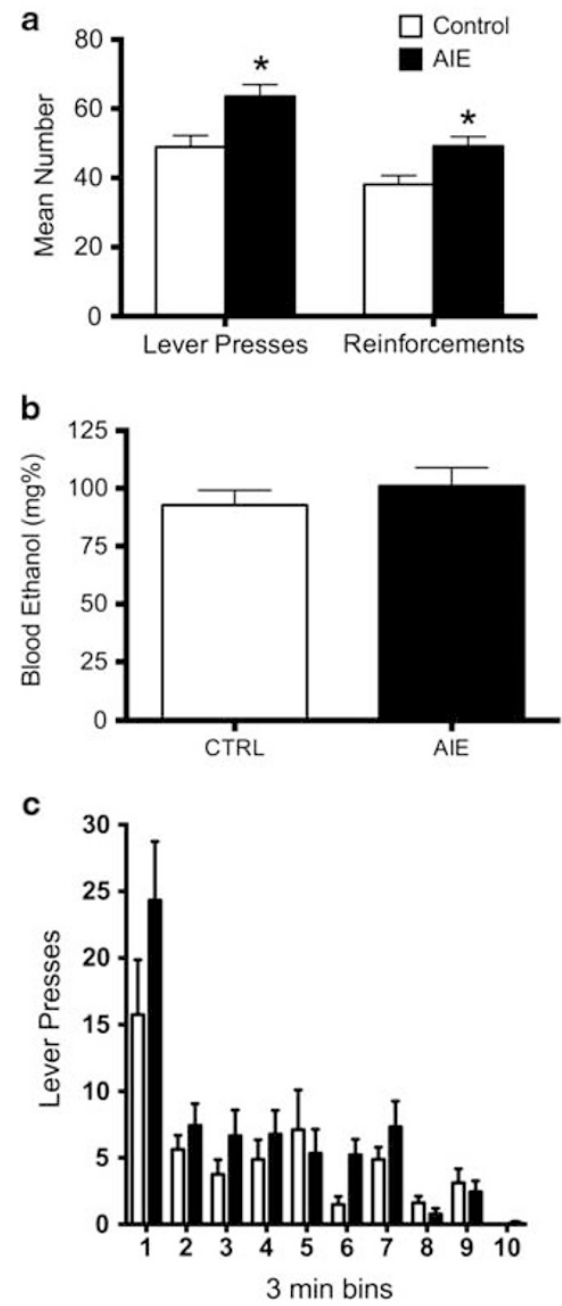

Figure 7 Exposure to AIE results in increased ethanol self-administration in adulthood. (a) Adult rats exposed to AlE showed both an increase in active lever responding for ethanol and ethanol reinforcements $(* p<0.05$ vs Control). (b) In spite of the greater number of ethanol reinforcements delivered, the BEC measured at the end of the operant session were not different. (c) While not statistically significant, there was a clear trend $(p=0.08)$ toward front loading of ethanol during the first 3 min of the selfadministration session in AlE-exposed rats compared with Control rats.
However, measurement of the BEC from tail vein blood at the end of the operant session revealed no significant difference in BEC between the Control and AIE groups (Figure $7 \mathrm{~b}$ ). This was an unexpected observation in light of the increased number of ethanol reinforcements that the AIE group received. While our operant apparatus did not include lickometers to measure the actual consumption of the ethanol reinforcement that was delivered, visual inspection of the reinforcer trough at the end of the 30min sessions revealed that all of the ethanol solution was being consumed. We therefore hypothesized that the lack of an observed difference in the BEC between the Control and AIE-exposed rats may have been impacted by the temporal differences in responding during the 30 -min sessions. When lever responding was divided into 3 -min time bins, it can be seen that most of the lever pressing occurred during the first $3 \mathrm{~min}$ of the operant session (Figure 7c). Furthermore, the enhanced lever pressing by the AIE-exposed group compared with Controls appeared to reflect front loading of consumption such that by the last 9 min of the session, they had greatly reduced lever pressing for ethanol. Although speculative, this may have resulted in a washout of the differences in BEC by the end of the sessions.

Extinction of drug seeking has been observed to represent new learning that has been shown to involve areas of the medial PFC. As our studies with set-shifting suggested that AIE exposure alters more complex forms of behavioral flexibility in the adult, we next examined whether AIE exposure affects extinction of ethanol self-administration. Following the establishment of stable levels of operant ethanol self-administration, rats were then subjected to daily sessions of extinction training. As shown in Figure 8, rats exposed to AIE had a significantly increased resistance to the extinction of ethanol-seeking behavior $[\mathrm{F}(42$, $60.01)=4.358, p<0.001]$. AIE rats showed increased active lever responding on multiple days of extinction relative to Control animals (Figure 8a, indicated by ${ }^{*} p$ values $<0.05$ ). AIE rats required significantly more extinction sessions to reach extinction criteria $[\mathrm{F}(3,33)=44.46, \quad p=0.0001]$ (Figure $8 \mathrm{~b}$, indicated by ${ }^{*} p=0.001$ ). As administration of the mGluR5 PAM CDPPB was observed to reverse AIE exposure-induced deficits in behavioral flexibility (Figure 5), we next examined the effects of CDPPB on extinction of ethanol self-administration. Consistent with the time course of extinction presented in Figure 7a, rats treated with CDPPB showed a significant reduction in active lever responding on multiple days of extinction compared with saline-treated Controls (Figure $8 \mathrm{a}$, indicated by ${ }^{\#} p$ values $<0.05$ ). In addition, rats treated with $\mathrm{CDPPB}$ required significantly fewer sessions to reach extinction criterion when compared with both Controls (Figure $8 \mathrm{~b}$, indicated by ${ }^{\#} p$ values $<0.001$ ) and AIE-treated rats (Figure $8 \mathrm{~b}$, indicated by ${ }^{* *} p$ values $\left.<0.001\right)$. In addition, not only was there a reversal of AIE-induced resistance to extinction, CDPPB administration to the Control group also enhanced the rate of extinction compared with the saline-injected Controls.

\section{DISCUSSION}

The current study demonstrates that repeated binge-like exposure to alcohol during early- to -middle adolescence 

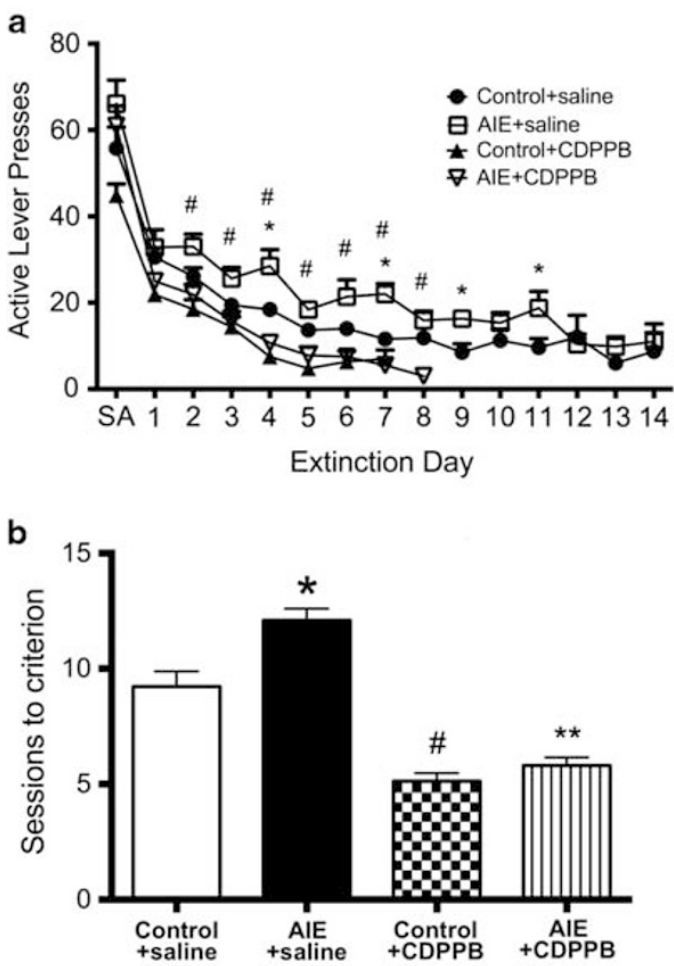

Figure 8 Exposure to AIE results in increased resistance to the extinction of ethanol-seeking behavior in adulthood. (a) Rats exposed to AIE displayed an increased resistance to extinction as evidenced by increased responding on the previously active lever on multiple days of extinction (* $p<0.05$ vs all other groups). Treatment with CDPPB prior to each extinction session attenuated the increased resistance to extinction observed in AlE-exposed rats and facilitated extinction in Control rats ( ${ }^{\#} p<0.05$ vs Control). (b) AIE exposure increased the number of trials required to reach extinction criteria ( ${ }^{p} p<0.05$ vs Control). Treatment with CDPPB prior to extinction training attenuated the increased resistance to extinction in AIE rats (** $p<0.05$ vs AIE) and reduced the number of sessions required for extinction in Control rats ( ${ }^{\#} p<0.05$ vs Control).

results in deficits in executive function in adulthood. It was observed that AIE-exposed adult rats exhibited poor flexibility when tested on a set-shifting task that requires the rats to alter their discrimination strategy following a change in the rule of the task. When tested on the EPM, AIE-exposed adult rats exhibited an increase in percent time and number of entries into the open arms of the maze, which may reflect a loss of inhibitory control. We also examined extinction of ethanol seeking, a simpler form of behavioral flexibility that involves new learning about changes in response/reward contingencies. Consistent with alterations in the function of this brain region, AIE-exposed adult rats exhibited resistance to extinction of ethanol seeking. Interestingly, administration of the putative cognitive-enhancing agent $\mathrm{CDPPB}$ prior to a strategy shift or extinction training completely reversed the AIE-induced cognitive deficits on these tasks. Finally, PLS-DA of DTI data obtained from fixed brains revealed reductions in the volume of several brain regions that are thought be important components of a distributed cognitive network of the PFC. Taken together, the results of the present study are consistent with the suggestion that alcohol abuse during adolescence results in alterations in the executive functioning in adulthood. These results further support the idea that cognitive enhancement therapy may be a promising approach to treat behavioral deficits associated with drug and alcohol abuse.

The ability to flexibly alter behavior in response to changing environmental contingencies is a core component of the executive control network. The set-shifting task utilized in the present study has been shown to be dependent upon the prelimbic (PrL) region of the medial PFC. Inactivation of this region does not impair learning of the initial rule of the task (eg, responding to the visual cue) but does impair the shift in response strategy to the new rule (eg, responding to position) (Floresco et al, 2008). In contrast, inactivation of the PrL does not impair the reversal of a response discrimination, nor does it affect the initial acquisition of simple discrimination rules. Thus, it has been suggested that the PrL cortex may have a privileged role in facilitating shifts between rules, strategies, and attentional sets (Birrell and Brown, 2000; Boulougouris et al, 2007; Floresco et al, 2008; Ragozzino et al, 1999). Our observation of a deficit in the ability of AIE-exposed adult rats to shift their response strategy on this task is consistent with the idea that early and excessive alcohol abuse can lead to an impairment in prefrontal functioning that facilitates cognitive flexibility. This being said, it is clear that complex behavior such as set-shifting are mediated by distributed neural circuits that incorporate other subcortical regions that are interconnected with the PFC, such as different regions of the ventral and dorsal striatum (Floresco et al, 2006; Haluk and Floresco, 2009; Ragozzino et al, 2002), and the medial thalamic nuclei (Block et al, 2007). Thus, it is possible that AIE may have also induced perturbation in the functioning of these other regions that contributed to the impairments in set-shifting observed here. It should be noted that unlike other regions such as the striatum, inactivation of the medial PFC induces a selective perseverative deficit in set-shifting when assessed by this approach (Floresco et al, 2008; Ragozzino et al, 1999). The fact that AIE rats tended to make more perseverative errors is consistent with the idea that pathophysiological mechanisms underlying impairments in flexibility were due, at least in part, to the disruption of normal PFC functioning.

In the initial set-shifting experiment, we observed a slight increase in the number of trial omissions made by AIE rats ( $\sim 4$ more omissions than controls over 80-100 trials). Thus, it is possible that this effect might reflect some type of non-specific disruption in the motivational processes. If this were the case, AIE would also be expected to increase omissions and impair learning during the initial visual cue discrimination, which had the same basic response requirements as the set-shift phase of the task. However, this was not the case as AIE-treated rats performed comparable to controls on this phase of the task. Moreover, in a second experiment, we failed to observe a significant effect on omissions during either the visual cue discrimination or the set-shift phase of the task. Furthermore, AIE rats displayed comparable breakpoints to Controls when lever pressing for reward on a PR schedule. Thus, if AIE did induce some form of motivational impairment, this is a relatively subtle and unreliable effect. On the other hand, we would argue that it is more likely that the impairments in 
behavioral flexibility induced by AIE are likely attributable primarily to disruption of the cognitive functioning that facilitate shifts between different discrimination strategies.

Clinical studies have indicated that adolescent alcohol abuse is associated with an increased propensity to engage in risky behavior, including experimentation with other drugs of abuse. Individuals who abuse alcohol during adolescence are at a significantly greater risk for developing an addiction in adulthood (Grant et al, 2001; Hasin and Glick, 1992). Although many of these clinical observations are confounded by the potential for a preexisting phenotype that predisposes the individual to drug and alcohol abuse, the abuse of alcohol during adolescence my produce developmental changes in the brain that result in enhanced susceptibility to addiction in adulthood. In the present study, we observed that AIE exposure led to an increase in response for alcohol during self-administration and an increase in alcohol-seeking behavior during extinction training when tested in adulthood. This result is consistent with other studies indicating that adolescent ethanol exposure enhances drinking in adulthood. For instance, repeated binge ethanol administration during adolescence was shown to enhance the voluntary consumption of a saccharin-sweetened ethanol solution in adult rats but did not affect saccharin only consumption (MaldonadoDevincci et al, 2010). A similar increase in drinking was also observed using an intermittent access paradigm in adolescent alcohol-exposed adult rats (Gilpin et al, 2012). Furthermore, a recent study revealed that early, but not late, adolescent ethanol exposure increased ethanol consumption in adulthood. Specifically, early adolescent exposure (PD30-PD43) increased ethanol intake and motivation to drink ethanol in adulthood, and these effects were associated with reduced aversive and increased rewarding properties of ethanol (Alaux-Cantin et al, 2013). However, other investigators reported that neither long-term voluntary ethanol exposure beginning in adolescence (Vetter et al, 2007) nor forced vapor exposure during the adolescent period (Slawecki and Betancourt, 2002) altered alcohol consumption in adulthood. Another study reported that binge ethanol exposure during early adolescence increased ethanol drinking during the latter part of adolescence, but did not alter drinking in adulthood (Fabio et al, 2013). The discrepancy among these studies could relate to experimental variables such as the method of adolescent ethanol exposure and the type of paradigm used to assess ethanol self-administration. In the present study, we observed increased response for ethanol and an increased number of ethanol reinforcements in the adult AIE-exposed rats, but no significant increase in the BEC was measured at the end of the operant session. Analysis of the time course of drinking during the session indicated that AIE-exposed rats respond more during the first $3 \mathrm{~min}$ of the $30 \mathrm{~min}$ session. While this difference did not reach statistical significance $(p=0.08$; Figure $5 c)$, this obvious trend is consistent with the suggestion that increased ethanol self-administration in the AIE-exposed rats during the initial part of the drinking session may have resulted in a 'leveling off of the BEC by the end of the session such that there was no longer a difference in drinking between the two groups at that time point. Another potential factor that may have had a role is that AIE-exposed adult rats metabolize alcohol at a faster rate, and thus, even though they self-administered more alcohol during the $30 \mathrm{~min}$ session, the BECs achieved were equivalent.

Of particular interest in our drinking studies was the observation that AIE-exposed rats took longer to extinguish cue-maintained ethanol-seeking behavior. If these results can be extrapolated to humans, an increase in resistance to extinction may indicate that not only does adolescent alcohol abuse promote greater alcohol seeking and consumption in the adult, but once drinking is initiated, it may be more difficult for these individuals to discontinue drinking, thus placing them at greater risk for the transition from controlled to uncontrolled drinking. In addition, these effects may also place such individuals at greater risk to relapse during periods of abstinence. The increased resistance to extinction of ethanol-seeking behavior in adult AIEexposed rats likely results from alterations in the PFC. Animal studies have shown the PrL cortex is a critical component in the circuitry of cocaine- and heroin-seeking behavior while the infralimbic (IfL) cortex is necessary for the expression of extinction behavior (for a review see (Gass and Chandler, 2013)). These converging lines of evidence suggest that the PrL cortex serves as an on-switch for conditioned reward seeking, whereas the IfL cortex functions as an off-switch for the expression of extinction behavior (Peters et al, 2009). Therefore, it is logical to suggest that adolescent ethanol exposure alters neural networks within the PFC that mediate the extinction of ethanolseeking behavior.

An important cognitive function of the PFC is the topdown inhibitory control of impulsive actions. The EPM is a task that is thought to represent a balance between the innate drive of the animal to explore a novel environment $v s$ fear/anxiety about that novel environment. In the present study, we observed that adult AIE-exposed rats exhibited more entries and spent more time in the open arms of the maze relative to the closed arms when compared with Control rats. This observation is in agreement with a recent study that also found an increase in the time spent in the open arms of the EPM in rats that had been exposed to alcohol during adolescence. As suggested by the authors of this study and in agreement with previous reports (Cruz et al, 1994; Fernandes and File, 1996; Pellow et al, 1985; Russell, 2011), this indicates that adult AIE-exposed rats have reduced passive anxiety-like behavior and/or increased impulsivity. In the present study, we were careful to minimize stress as an experimental confound. This included during rearing (eg, daily handling from weaning until PD42, passive exposure to ethanol using vapor inhalation and pair-housing with littermates) and during behavioral testing. In addition, the rats we tested on the EPM had undergone prior training and testing on the operant setshifting task, and thus been well acclimated to the stress of handling and experimental manipulations. Furthermore, using the baseline conditions reported in the Gilpin et al (2012) study, we adjusted the testing conditions of the EPM so that Control rats spent $\sim 35-40 \%$ of their time in the open arms. These experimental conditions allow the rats to alter the time spent in the closed $v s$ open arms in such a way that it is not biased toward one or the other by the testing conditions. We suggest that the observation of increases in entries and time spent in the open arms is consistent with 
an increase in impulsivity. This is also consistent with a recent report using a modified open field conflict task in which it was observed that adult AIE-exposed rats displayed more disinhibitory behavior compared with non-alcoholexposed Control rats (Ehlers et al, 2013a). As with the EPM, the possibility that the rats were exhibiting less anxiety-like behavior on this task cannot be excluded. However, we are not aware on any previous studies demonstrating that alcohol abuse is associated with reduction in anxiety following extended abstinence, and thus, the most logical interpretation of the data is that AIE exposure results in disinhibition of exploratory behavior in adulthood. Furthermore, this disinhibition may relate to the impairment of PFC function. Support for this notion comes from the observation that inactivation of the PFC increases the proportion of time spent on the open arms of an EPM (Shah et al, 2004) in a manner similar to the AIE-exposed rats.

As an initial investigation of the effects of AIE exposure on brain structure in adulthood, we conducted DTI of the intact heads of a subset of rats following the completion of behavioral testing (EPM and set-shifting). After obtaining volumetric measurements of the whole brain and 32 different brain regions, we then analyzed the data using a multivariate modeling approach to identify group membership by projecting predicted and observable variables into new space (as opposed to hyperplane space in principal component regression). This analysis revealed a group of five brain regions in the AIE-exposed groups that significantly contributed to the model. Four of these areas (hippocampus, dorsal striatum, thalamus, and neocortex) were reduced in volume, whereas the hypothalamus was increased in volume in the AIE vs Control rats. While these studies are exploratory and follow-up histological studies are needed, the results of this approach may have identified a group of brain regions whose structure was pathologically altered in the AIE-exposed adult. Interestingly, these brain regions have been implicated in various aspects of cognition and addiction, and a reasonable prediction is that these structural changes will negatively impact their functional connectivity.

An additional novel finding in the present study was that administration of a PAM of mGluR5 reversed the behavioral deficits in adult rats that had been exposed to alcohol during adolescence. Agents that act as PAMs of mGluR5 have been reported to exhibit procognitive properties that may relate to actions within the distributed neural networks of the PFC that regulate executive function (Ayala et al, 2009; Fowler et al, 2013; Kroker et al, 2011; Xu et al, 2013). A facilitatory action on executive control is consistent with our observation that CDPPB can ameliorate impairments in behavioral flexibility. In addition, extinction of fear and drug memories represents a simpler form of flexibility entailing the formation of a new memory that competes with the original drug or fear memory. In agreement with previous studies showing that $\mathrm{CDPPB}$ can facilitate the extinction of cocaine memories (Cleva et al, 2011; Gass and Olive, 2009), we observed that CDPPB facilitates the extinction of ethanol self-administration, including the reversal of resistance to extinction observed in adult AIEexposed rats. In light of the fact that both set-shifting and extinction are critically dependent on intact medial PFC function, our results suggest that the procognitive actions of
CDPPB relate, at least in part, to actions within the $\mathrm{PFC}$ that enhance cognitive control of behavior. Importantly, the ability of CDPPB to reverse behavioral deficits induced by AIE does not appear to result from alterations in locomotor activity as previous research in our laboratory (Cleva et al, 2011) and others (Kufahl et al, 2012; Reichel et al, 2011) has shown that this dose of CDPPB does not alter motor behavior.

Although the processes by which activation of mGluR5 receptors enhance cognition are not clear, one potential mechanism may be through indirect actions on NMDA receptors. Blockade of the NMDA receptor has been shown to significantly impair performance in an operant setshifting task likely through mechanisms within the mPFC (Stefani et al, 2003; Stefani and Moghaddam, 2005). Furthermore, this impairment is reversed when CDPPB is administered immediately after administration of the NMDA antagonist MK801 (Darrah et al, 2008). Taken together with the results of the present study, these observations strongly suggest that modulation of mGluR5 can reduce impairments in behavioral flexibility possibly through actions in the mPFC. However, additional mechanisms by which mGluR5 could influence cognition include direct actions of the receptors on neuronal activity as well as modulation of glutamatergic neurotransmission by astroglia. For example, in mice, it has been shown that a 30 -day history of binge alcohol exposure leads to an increase in mGluR5, Homer2, and NR2B in the nucleus accumbens shell region and that mGluR5/Homer2 signaling within this region is important in mediating high levels of alcohol intake (Cozzoli et al, 2012). Similarly, a clinical study has shown that mGluR1/5 receptors are increased in the CA2 region of the hippocampus in alcoholics (Kupila et al, 2013). However, the effect of adolescent alcohol exposure on mGluR5 expression and the resulting consequences on adult cognitive behavior remain to be determined. Regardless of the exact mechanism(s), our findings provide further evidence that PAMs of mGluR5 receptors represent a promising therapeutic approach for the treatment of cognitive dysfunction, including deficits observed in adults who abuse alcohol during adolescence.

The results of the present study, together with reports from others, suggest that adolescent alcohol abuse is associated with deficits in executive function and alterations in behavioral control in adulthood. Although not examined in the present study, it has been shown that exposure to ethanol during the adolescent period results in increased risky behavior in adulthood (Clark et al, 2012; Nasrallah et al, 2011; Nasrallah et al, 2009). Interestingly, Akil and colleagues have shown that rats selectively bred as 'highresponders' (bHR) show similar characteristics associated with prefrontal control of behavior (Flagel et al, 2013). For instance, compared with low-bred responders (bLR), bHR rats seek stimuli associated with rewards and exhibit behavioral disinhibition, increased exploratory behavior, a greater propensity for relapse to drug taking, and increased risk taking on a probabilistic decision-making task, and it has been suggested that the bHR phenotype parallels certain aspects of externalizing disorders in humans. Externalizing disorders have been linked to cortical dysfunction and increased risk for the development of drug and alcohol problems (Hulvershorn et al, 2013). Although speculative, 
an interesting idea that appears to be supported by studies in animal models is that adolescent alcohol exposure promotes development of a behavioral phenotype in adulthood that is similar to the phenotype of externalizing disorders that are often observed in at-risk adolescent individuals.

\section{FUNDING AND DISCLOSURE}

The authors declare no conflict of interest.

\section{ACKNOWLEDGEMENTS}

This work is funded by grants U01AA019967, U24AA020023, U24AA020024, P50AA010761, R01AA010983, K99AA020537, F32AA021642, CIHR MOP102478, P30CA016086-35-37, and U54CA151652-01-04.

\section{REFERENCES}

Abernathy K, Chandler LJ, Woodward JJ (2010). Alcohol and the prefrontal cortex. Int Rev Neurobiol 91: 289-320.

Alaux-Cantin S, Warnault V, Legastelois R, Botia B, Pierrefiche O, Vilpoux $C$ et al (2013). Alcohol intoxications during adolescence increase motivation for alcohol in adult rats and induce neuroadaptations in the nucleus accumbens. Neuropharmacology 67: 521-531.

Ayala JE, Chen Y, Banko JL, Sheffler DJ, Williams R, Telk AN et al (2009). mGluR5 positive allosteric modulators facilitate both hippocampal LTP and LTD and enhance spatial learning. Neuropsychopharmacology 34: 2057-2071.

Birrell JM, Brown VJ (2000). Medial frontal cortex mediates perceptual attentional set shifting in the rat. J Neurosci 20: 4320-4324.

Blakemore SJ, Robbins TW (2012). Decision-making in the adolescent brain. Nat Neurosci 15: 1184-1191.

Block AE, Dhanji H, Thompson-Tardif SF, Floresco SB (2007). Thalamic-prefrontal cortical-ventral striatal circuitry mediates dissociable components of strategy set shifting. Cereb Cortex 17: 1625-1636.

Boulougouris V, Dalley JW, Robbins TW (2007). Effects of orbitofrontal, infralimbic and prelimbic cortical lesions on serial spatial reversal learning in the rat. Behav Brain Res 179: 219-228.

Casey BJ, Jones RM (2010). Neurobiology of the adolescent brain and behavior: implications for substance use disorders. J Am Acad Child Adolesc Psychiatry 49: 1189-1201 quiz 1285.

Chambers RA, Taylor JR, Potenza MN (2003). Developmental neurocircuitry of motivation in adolescence: a critical period of addiction vulnerability. Am J Psychiatry 160: 1041-1052.

Clark JJ, Nasrallah NA, Hart AS, Collins AL, Bernstein IL, Phillips PE (2012). Altered risk-based decision making following adolescent alcohol use results from an imbalance in reinforcement learning in rats. PLoS One 7: e37357.

Cleva RM, Hicks MP, Gass JT, Wischerath KC, Plasters ET, Widholm JJ et al (2011). mGluR5 positive allosteric modulation enhances extinction learning following cocaine self-administration. Behav Neurosci 125: 10-19.

Cozzoli DK, Courson J, Caruana AL, Miller BW, Greentree DI, Thompson AB et al (2012). Nucleus accumbens mGluR5associated signaling regulates binge alcohol drinking under drinking-in-the-dark procedures. Alcohol Clin Exp Res 36: 1623-1633.

Crews FT, Nixon K, Wilkie ME (2004). Exercise reverses ethanol inhibition of neural stem cell proliferation. Alcohol 33: 63-71.
Cruz AP, Frei F, Graeff FG (1994). Ethopharmacological analysis of rat behavior on the elevated plus-maze. Pharmacol Biochem Behav 49: 171-176.

Darrah JM, Stefani MR, Moghaddam B (2008). Interaction of $\mathrm{N}$-methyl-D-aspartate and group 5 metabotropic glutamate receptors on behavioral flexibility using a novel operant setshift paradigm. Behav Pharmacol 19: 225-234.

Ehlers CL, Liu W, Wills DN, Crews FT (2013a). Periadolescent ethanol vapor exposure persistently reduces measures of hippocampal neurogenesis that are associated with behavioral outcomes in adulthood. Neuroscience 244: 1-15.

Ehlers CL, Oguz I, Budin F, Wills DN, Crews FT (2013b). Periadolescent ethanol vapor exposure produces reductions in hippocampal volume that are correlated with deficits in prepulse inhibition of the startle. Alcohol Clin Exp Res 37: 1466-1475.

Enomoto T, Tse MT, Floresco SB (2011). Reducing prefrontal gamma-aminobutyric acid activity induces cognitive, behavioral, and dopaminergic abnormalities that resemble schizophrenia. Biol Psychiatry 69: 432-441.

Fabio MC, Nizhnikov ME, Spear NE, Pautassi RM (2013). Binge ethanol intoxication heightens subsequent ethanol intake in adolescent, but not adult, rats. Dev Psychobiol 56: 574-583.

Fernandes C, File SE (1996). The influence of open arm ledges and maze experience in the elevated plus-maze. Pharmacol Biochem Behav 54: 31-40.

Flagel SB, Waselus M, Clinton SM, Watson SJ, Akil H (2013). Antecedents and consequences of drug abuse in rats selectively bred for high and low response to novelty. Neuropharmacology 76 Pt B: $425-436$.

Floresco SB, Block AE, Tse MT (2008). Inactivation of the medial prefrontal cortex of the rat impairs strategy set-shifting, but not reversal learning, using a novel, automated procedure. Behav Brain Res 190: 85-96.

Floresco SB, Ghods-Sharifi S, Vexelman C, Magyar O (2006). Dissociable roles for the nucleus accumbens core and shell in regulating set shifting. $J$ Neurosci 26: 2449-2457.

Fowler SW, Walker JM, Klakotskaia D, Will MJ, Serfozo P, Simonyi A et al (2013). Effects of a metabotropic glutamate receptor 5 positive allosteric modulator, $\mathrm{CDPPB}$, on spatial learning task performance in rodents. Neurobiol Learn Mem 99: 25-31.

Gass JT, Chandler LJ (2013). The plasticity of extinction: contribution of the prefrontal cortex in treating addiction through inhibitory learning. Front Psychiatry 4: 46.

Gass JT, Olive MF (2009). Positive allosteric modulation of mGluR5 receptors facilitates extinction of a cocaine contextual memory. Biol Psychiatry 65: 717-720.

Gilpin NW, Karanikas CA, Richardson HN (2012). Adolescent binge drinking leads to changes in alcohol drinking, anxiety, and amygdalar corticotropin releasing factor cells in adulthood in male rats. PLoS One 7: e31466.

Grant BF, Stinson FS, Harford TC (2001). Age at onset of alcohol use and DSM-IV alcohol abuse and dependence: a 12-year follow-up. J Subst Abuse 13: 493-504.

Haluk DM, Floresco SB (2009). Ventral striatal dopamine modulation of different forms of behavioral flexibility. Neuropsychopharmacology 34: 2041-2052.

Hasin DS, Glick H (1992). Severity of DSM-III-R alcohol dependence: United States, 1988. Br J Addict 87: 1725-1730.

Horio M, Fujita Y, Hashimoto K (2013). Therapeutic effects of metabotropic glutamate receptor 5 positive allosteric modulator CDPPB on phencyclidine-induced cognitive deficits in mice. Fundam Clin Pharmacol 27: 483-488.

Hulvershorn LA, Finn P, Hummer TA, Leibenluft E, Ball B, Gichina $\mathrm{V}$ et al (2013). Cortical activation deficits during facial emotion processing in youth at high risk for the development of substance use disorders. Drug Alcohol Depend 131: 230-237.

Kroker KS, Rast G, Rosenbrock H (2011). Differential effect of the mGlu5 receptor positive allosteric modulator ADX-47273 on 
early and late hippocampal LTP. Neuropharmacology 61: 707-714.

Kufahl PR, Hood LE, Nemirovsky NE, Barabas P, Halstengard C, Villa A et al (2012). Positive allosteric modulation of mGluR5 accelerates extinction learning but not relearning following methamphetamine self-administration. Front Pharmacol 3: 194.

Kupila J, Karkkainen O, Laukkanen V, Tupala E, Tiihonen J, Storvik M (2013). mGluR1/5 receptor densities in the brains of alcoholic subjects: a whole-hemisphere autoradiography study. Psychiatry Res 212: 245-250.

Maldonado-Devincci AM, Alipour KK, Michael LA, Kirstein CL (2010). Repeated binge ethanol administration during adolescence enhances voluntary sweetened ethanol intake in young adulthood in male and female rats. Pharmacol Biochem Behav 96: 476-487.

McQueeny T, Schweinsburg BC, Schweinsburg AD, Jacobus J, Bava $S$, Frank LR et al (2009). Altered white matter integrity in adolescent binge drinkers. Alcohol Clin Exp Res 33: 1278-1285.

Nasrallah NA, Clark JJ, Collins AL, Akers CA, Phillips PE, Bernstein IL (2011). Risk preference following adolescent alcohol use is associated with corrupted encoding of costs but not rewards by mesolimbic dopamine. Proc Natl Acad Sci USA 108: 5466-5471.

Nasrallah NA, Yang TW, Bernstein IL (2009). Long-term risk preference and suboptimal decision making following adolescent alcohol use. Proc Natl Acad Sci USA 106: 17600-17604.

Nixon K, Crews FT (2002). Binge ethanol exposure decreases neurogenesis in adult rat hippocampus. J Neurochem 83: 1087-1093.

Paxinos G, Watson C (2005). The Rat Brain in Stereotaxic Coordinates. 5th edn. Academic Press: San Diego.

Pellow S, Chopin P, File SE, Briley M (1985). Validation of open:closed arm entries in an elevated plus-maze as a measure of anxiety in the rat. J Neurosci Methods 14: 149-167.

Petanjek Z, Judas M, Simic G, Rasin MR, Uylings HB, Rakic P et al (2011). Extraordinary neoteny of synaptic spines in the human prefrontal cortex. Proc Natl Acad Sci USA 108: 13281-13286.

Peters J, Kalivas PW, Quirk GJ (2009). Extinction circuits for fear and addiction overlap in prefrontal cortex. Learn Mem 16: 279-288.

Ragozzino ME, Detrick S, Kesner RP (1999). Involvement of the prelimbic-infralimbic areas of the rodent prefrontal cortex in behavioral flexibility for place and response learning. J Neurosci 19: 4585-4594.

Ragozzino ME, Ragozzino KE, Mizumori SJ, Kesner RP (2002). Role of the dorsomedial striatum in behavioral flexibility for response and visual cue discrimination learning. Behav Neurosci 116: 105-115.

Rakic P, Bourgeois JP, Goldman-Rakic PS (1994). Synaptic development of the cerebral cortex: implications for learning, memory, and mental illness. Prog Brain Res 102: 227-243.

Ranaldi R, Roberts DC (1996). Initiation, maintenance and extinction of cocaine self-administration with and without conditioned reward. Psychopharmacology (Berl) 128: 89-96.

Reichel CM, Schwendt M, McGinty JF, Olive MF, See RE (2011). Loss of object recognition memory produced by extended access to methamphetamine self-administration is reversed by positive allosteric modulation of metabotropic glutamate receptor 5 . Neuropsychopharmacology 36: 782-792.

Richardson NR, Roberts DC (1996). Progressive ratio schedules in drug self-administration studies in rats: a method to evaluate reinforcing efficacy. J Neurosci Methods 66: 1-11.

Rumple A, McMurray M, Johns J, Lauder J, Makam P, Radcliffe M et al (2013). 3-dimensional diffusion tensor imaging (DTI) atlas of the rat brain. PLoS One 8: e67334.

Russell VA (2011). Overview of animal models of attention deficit hyperactivity disorder (ADHD). Curr Protoc Neurosci Chapter 9: Unit 935.

Shah AA, Sjovold T, Treit D (2004). Inactivation of the medial prefrontal cortex with the GABAA receptor agonist muscimol increases open-arm activity in the elevated plus-maze and attenuates shock-probe burying in rats. Brain Res 1028: 112-115.

Simms JA, Bito-Onon JJ, Chatterjee S, Bartlett SE (2010). LongEvans rats acquire operant self-administration of $20 \%$ ethanol without sucrose fading. Neuropsychopharmacology 35: 1453-1463.

Simms JA, Richards JK, Mill D, Kanholm I, Holgate JY, Bartlett SE (2011). Induction of multiple reinstatements of ethanol- and sucrose-seeking behavior in Long-Evans rats by the alpha-2 adrenoreceptor antagonist yohimbine. Psychopharmacology (Berl) 218: 101-110.

Slawecki CJ, Betancourt M (2002). Effects of adolescent ethanol exposure on ethanol consumption in adult rats. Alcohol 26: 23-30.

Spear LP (2000). The adolescent brain and age-related behavioral manifestations. Neurosci Biobehav Rev 24: 417-463.

Stefani MR, Groth K, Moghaddam B (2003). Glutamate receptors in the rat medial prefrontal cortex regulate set-shifting ability. Behav Neurosci 117: 728-737.

Stefani MR, Moghaddam B (2005). Systemic and prefrontal cortical NMDA receptor blockade differentially affect discrimination learning and set-shift ability in rats. Behav Neurosci 119: 420-428.

Stefani MR, Moghaddam B (2010). Activation of type 5 metabotropic glutamate receptors attenuates deficits in cognitive flexibility induced by NMDA receptor blockade. Eur J Pharmacol 639: 26-32.

Uslaner JM, Parmentier-Batteur S, Flick RB, Surles NO, Lam JS, McNaughton CH et al (2009). Dose-dependent effect of CDPPB, the mGluR5 positive allosteric modulator, on recognition memory is associated with GluR1 and CREB phosphorylation in the prefrontal cortex and hippocampus. Neuropharmacology 57: $531-538$

Vetter CS, Doremus-Fitzwater TL, Spear LP (2007). Time course of elevated ethanol intake in adolescent relative to adult rats under continuous, voluntary-access conditions. Alcohol Clin Exp Res 31: $1159-1168$.

Witt ED (2010). Research on alcohol and adolescent brain development: opportunities and future directions. Alcohol 44: 119-124.

Xu J, Zhu Y, Kraniotis S, He Q, Marshall JJ, Nomura T et al (2013). Potentiating mGluR5 function with a positive allosteric modulator enhances adaptive learning. Learn Mem 20: 438-445.

Supplementary Information accompanies the paper on the Neuropsychopharmacology website (http://www.nature.com/npp) 